\title{
Statins and non-alcoholic fatty liver disease
}

\section{Dear Editor,}

In April 9 issue, van den Berg et al ${ }^{1}$ report interesting results on the indication for lipid-lowering treatment in a large cohort with suspected non-alcoholic fatty liver disease (NAFLD) within the population-based Lifelines Cohort Study. Fatty liver index (FLI) $\geq 60$ was used as a proxy of NAFLD and the NAFLD fibrosis score (NFS) to identify the NAFLD patients with suspected advanced fibrosis. Cardiovascular disease (CVD) risk was established by the 2016 European society of Cardiology/European Atherosclerosis Society (ESC/EAS) Guidelines for the Management of Dyslipidemias. ${ }^{2}$

Subjects with FLI $\geq 60$ (suspected NAFLD) had an increased 10year predicted cardiovascular risk compared to those with $\mathrm{FLI}<60$ with an approximately 2 times higher need for statin therapy based on CVD risk prediction and their LDL cholesterol level. Subjects with a FLI $\geq 60$ were more likely to be classified with type 2 diabetes, Metabolic Syndrome (MetS), history of CVD and impaired renal function. Interestingly, estimated 10-year very high cardiovascular risk was approximately 4 times higher in subjects with a NFS $>0.676$ compared to those with the absence of advanced fibrosis. Finally, indication for statin treatment was positively associated with a FLI $\geq 60$ after controlling for age, sex, current smoking, impaired renal function, and the presence of MetS and its individual components. The above results have an even greater relevance if we consider that all the subjects who were already on statin therapy were subtracted from the analysis.

These findings may have an important clinical relevance and emphasize the need for effective treatment with statins in patients with NAFLD. Indeed, accumulating evidence suggests that CVD, rather than liver disease, dictates the outcomes in NAFLD. ${ }^{3}$ Besides, in most subjects NAFLD constitutes the hepatic component of MetS and numerous patients have atherogenic dyslipidemia.

This study further supports the results of a previous study by our group where under prescription of statins in patients with NAFLD was observed. ${ }^{4}$ In fact, mild liver enzyme elevation remains a concern and despite its proven efficacy and safety, ${ }^{5}$ statin administration is sometimes limited by the worry about related side effects. Indeed, there is a tendency of general physicians to discourage statin use in patients with baseline elevation of serum liver enzymes and/ or to discontinue medication when minor alterations were appreciated. Of note, in our study, statin under-use was high also in patients at very high $\mathrm{CV}$ risk such as those with a previous $\mathrm{CV}$ event.

This study by van den Berg et al further stresses the issue of under prescription of statins in people with NAFLD and indication for treatment, based on $\mathrm{CV}$ risk class and low-density lipoprotein cholesterol target according to ESC/EAS guidelines.

\section{CONFLICT OF INTEREST}

The authors do not have any disclosures to report.

$$
\begin{array}{r}
\text { Francesco Angelico }{ }^{1} \text { (D) } \\
\text { Francesco Baratta } \\
\text { Daniele Pastori } \\
\text { Maria Del Ben }
\end{array}
$$

${ }^{1}$ Department of Public Health and Infectious Diseases, Sapienza University of Rome, Rome, Italy

${ }^{2}$ Department of Internal Medicine and Medical Specialties, I Clinica Medica, Sapienza University of Rome, Rome, Italy

Correspondence

Francesco Angelico, Department of Public Health and Infectious Diseases, Sapienza University of Rome, Rome, Italy. Email: Francesco.angelico@uniroma1.it

\section{ORCID}

Francesco Angelico (iD) https://orcid.org/0000-0002-9372-3923

Francesco Baratta (D) https://orcid.org/0000-0003-1708-272X

Daniele Pastori (D) https://orcid.org/0000-0001-6357-5213

Maria Del Ben (D) https://orcid.org/0000-0003-1199-8454

\section{REFERENCES}

1. van den Berg EH, Wolters A, Dullaart R, et al. Prescription of statins in suspected non-alcoholic fatty liver disease and high cardiovascular risk, a population-based study. Liver Int. 2019. https://doi.org/ 10.1111/liv.14116

2. Catapano AL, Graham I, De Backer G, et al. 2016 ESC/EAS guidelines for the management of dyslipidaemias: the Task Force for the Management of Dyslipidaemias of the European Society of Cardiology (ESC) and European Atherosclerosis Society (EAS) Developed with the special contribution of the European Association for Cardiovascular Prevention Rehabilitation (EACPR). Atherosclerosis. 2016;253:281-344. https://doi.org/10.1016/j.atherosclerosis.2016.08.018

3. Del Ben M, Baratta F, Polimeni L, Angelico F. Non-alcoholic fatty liver disease and cardiovascular disease: epidemiological, clinical and pathophysiological evidences. Int Emerg Med. 2012;7(Suppl 3):S291-S296. https://doi.org/10.1007/s11739-012-0819-4

4. Del Ben M, Baratta F, Polimeni L, et al. Under-prescription of statins in patients with non-alcoholic fatty liver disease. Nutr Metab Cardiovasc Dis. 2017;27:161-167. https://doi.org/10.1016/j.numecd.2016.09.011

5. Pastori D, Polimeni L, Baratta F, Pani A, Del Ben M, Angelico F. The efficacy and safety of statins for the treatment of non-alcoholic fatty liver disease. Dig Liver Dis. 2015;47:4-11. https://doi.org/10.1016/ j.dld.2014.07.170 